its name, nearly $30 \%$ of patients report no recognisable rat bite. Precise history-taking related to contact with rats and other rodents and detection of skin eruptions can be clues to diagnosis of this infection.

\section{WORKPLACE INFLUENZA: TO BE VACCINATED OR NOT TO BE VACCINATED, THAT'S THE QUESTION FOR HEALTHCARE WORKERS - THE ETHICS-}

WLC Van Hooste*. MD, Mediwet Occupational Health Services, External Service for Prevention and Protection at Work, Ghent, Belgium

\subsection{6/oemed-2018-ICOHabstracts.601}

Introduction Seasonal influenza (SI) vaccination has been recommended for healthcare workers (HCWs) for many years. Despite decades of efforts to encourage HCWs to be immunised, vaccination uptake levels remain low. Most voluntary policies to increase vaccination rates among HCWs have not been very effective. How to close the gap between desired and current vaccination rates, that's the question. Whether (semi)mandatory vaccination policies (e.g., vaccination-required, vaccinate-or-mask, 'may not work during facility outbreaks' policies) are justified is an ethical issue.

Methods We explored the ethical, moral, empirical and evidential arguments for or against (mandatory) SI vaccination of HCWs in the literature (2011-2017). Neither the 'clinical' nor the 'public health' ethics frameworks resolve the question fully. Are the 'medical ethical principles' of beneficence (an action done to benefit others) and non-maleficence (one ought not to inflict evil or harm) sufficient enough to support mandatory influenza vaccination policies?

Results Recently 'components of justice' frameworks were also added to the ungoing ethical debate. HCWs remain bound by ethical principles of both beneficence and non-maleficence. It is very unlikely that purely voluntary programmes will achieve vaccination rates among HCWs that are sufficient to meet the ethical obligations of beneficence and non-maleficence. Although there is increasing evidence, that individuals are more inclined to get vaccinated if this benefits other, if their own costs are low.

Further studies documenting the impact of HCWs influenza vaccination outcomes on the patients' health, would inform decisions on the use of mandatory vaccine policies in HCWs. Despite the ongoing debate about the evidence, some governments and health care organisations have judged that the scientific evidence is sufficiently strong to justify (semi)mandatory vaccination policies for HCWs.

Discussion Garning early and broad-based support from employers, employees, governmental administration, human resources, and occupational health appears to be important for development of a SI vaccine mandate.

\section{WORKPLACE MEASLES: RISK ANALYSIS \& MATERNITY PROTECTION - A REVIEW OF LITERATURE}

WLC Van Hooste*. MD, Mediwet Occupational Health Services, External Service for Prevention and Protection at Work, Ghent, Belgium

10.1136/oemed-2018-ICOHabstracts.602
Introduction Measles is one of the most transmissible and highly communicable human infectious diseases known. It has a $90 \%$ secondary attack rate and the reproduction number is around 17. It is probably the most deadly of all childhood rash/fever illnesses. Since 2008 a rise in measles cases has been reported with outbreaks in 36 European countries.

Methods A review of literature generated 85 articles of interest and were consulted for this review.

Result Risk analysis shows that schools play a crucial role in the transmission chain of measles. At highest risk are infants under one year of age, adolescents and young adults. The risk is the highest in the part of the population that typically constitutes the engine of airborne transmission through social contacts at childcare, school, and within and between households. A catch-up campaign focusing all young adults and school personnel is necessary to achieve elimination in the short term. Health care workers (HCWs) are at substantially higher risk (2-13 times) than the general population for becoming infected with measles. Waning immunity in these HCWs will increase the risk of nosocomial infections. Nosocomial outbreaks are also exacerbated by atypical presentations of the disease.

Pregnant women with measles had significantly higher risks of adverse maternal, fetal, and neonatal outcomes like sudden spontaneous abortions, stillbirth, early fetal death, and premature deliveries. Measles in pregnancy is complicated by a high maternal morbidity and mortality rate.

Discussion To achieve 'herd immunity', more than 95\% of the community must be vaccinated with 2 doses. For those unprotected, in doubt about clinical measles in anamnesis, in doubt about vaccination status or undocumented vaccination with 2 doses of measles containing vaccination, it is necessary to provide two doses with an interval of one month. Special focus on young adults among kindergarten, (pre)school personnel and HCWs is necessary.

\section{INFLUENZA INFECTION AND IMMUNISATION COVERAGE AMONG HEALTHCARE PROFESSIONALS IN NATIONAL GUARD HOSPITAL, WESTERN SAUDI ARABIA}

${ }^{1,2} \mathrm{FM}$ Farahat*, ${ }^{1,2} \mathrm{~A}$ AlSaedi, ${ }^{2} \mathrm{MF}$ AbdulAziz. ${ }^{1}$ King Saud bin AbdulAziz University for Health Sciences, Jeddah, Saudi Arabia; ${ }^{2}$ King AbdulAziz Medical City, Jeddah, Saudi Arabia

\subsection{6/oemed-2018-ICOHabstracts.603}

Introduction Burden of influenza infection among healthcare professionals (HCPs) is high including transmission to high risk and severely-ill patients. This chart review aims is to record laboratory confirmed influenza cases and influenza immunisation coverage among HCPs in Ministry of National Guard hospital, a 500-bed tertiary care hospital, in western Saudi Arabia.

Methods Laboratory confirmed influenza cases among healthcare professionals from June 2014 - June 2017 were reviewed. Influenza immunisation and reasons of declining to receive the vaccine were recorded.

Results During the study period, 78 HCPs had laboratory confirmed influenza $(9.1 \%$ of all confirmed cases). There were 18 HCPs with H1N1 (8.1\%), 43 with influenza A (11.1\%) and 17 with influenza B (6.8\%). In the meantime, influenza immunisation coverage ranged from 33\%-64\% among physicians, 80\%-93\% among nurses and 32\%-41\% among paramedicals 
(i.e., medical technicians, pharmacists and administrators). Reasons of declining influenza vaccination included doubt of the need for the vaccine $(27.8 \%)$, concern of vaccine safety (21.0\%), bad experience following vaccination in the previous year $(41.5 \%)$, insufficient time to attend immunisation clinic $(9.6 \%)$ and fear of the needle $(6.6 \%)$.

Conclusion Number of confirmed influenza cases is mostly under-reported. HCPs are at increased risk for influenza infection and efforts should focus on Infection prevention strategies and increasing immunisation coverage among HCPs and patients.

\section{OCCUPATIONAL HEALTH PRACTICES FOR WASTEWATER TREATMENT WORKERS}

1,2S Porru, ${ }^{2,3} \mathrm{~A}$ Carta, ${ }^{3} \mathrm{~B}$ Bellina, ${ }^{2,3} \mathrm{C}$ Arici*. ${ }^{1}$ Dept. of Diagnostics and Public Health, University of Verona, Verona, Italy; 'University Research Centre 'MISTRAL', University of Brescia, Brescia, Italy; ${ }^{3}$ Dept. of Medical-Surgical Specialties, Radiological Sciences and Public Health, University of Brescia, Brescia, Italy

\subsection{6/oemed-2018-ICOHabstracts.604}

Introduction The increase in the global population entails large production of wastewaters (WW) around the world. WW treatment plants (WWTP) may represent a challenging and hazardous work environment, since various biological agents (viruses, bacteria, fungi, parasites), as well as endotoxins, can be transmitted to the ambient air in WW bioaerosols and cause various disorders. No occupational exposure limit values are currently available. Thus, the potential biohazard $(\mathrm{BH})$ to WWTP workers (WWTPW) has become an increasingly relevant occupational health and safety (OHS) issue. Aims of our contribution are: to provide a state-of-the-art overview on the occupational $\mathrm{BH}$ to WWTPW and to elaborate good OHS practices, based on a field study.

Methods Ten-year (2008-2017) scoping review of articles in the PubMed ${ }^{\circledast}$ database, published in English, French, Italian or Spanish; full text review of the articles. Field study in a sample of 4 WWTP companies managing more than 200 plants overall, with a capacity varying from less than 1000 to more than 870000 population equivalents and employing about 5 to 50 WWTPW. The study entails: collection of OHS documentation on risk assessment and management, health surveillance, education and training; microbiological environmental monitoring (culturable, countable, assayable biological contaminants); investigation on general and specific health data in a sample of WWTPW, by administering a questionnaire regarding socio-demographic aspects, life habits, occupational and clinical history, preventive and protective measures, and by collecting some functional data (e.g. respiratory function); exploring correlations between $\mathrm{BH}$ and $\mathrm{OHS}$ outcomes among WWTPW.

Result Scoping review is ongoing as well as enrolment of WWTP and occupational data collection.

Discussion Main expected results are: assessment of occupational BH in WWTP; development of good OHS practices, particularly on risk assessment, health surveillance, fitness for work, immunisation and preventive practices in WWTP; generation of benchmarks; contribution to the research on occupational limit values for $\mathrm{BH}$.

\section{3 AIRBORNE DISPERSION OF LEPTOSPIROSIS IN A MEAT PROCESSING PLANT}

${ }^{1}$ DJ McLean*, ${ }^{2} \mathrm{M}$ leblanc-Maridor, ${ }^{3} \mathrm{RJ} H a l l,{ }^{3} \mathrm{NE}$ Moore, ${ }^{3} \mathrm{~K}$ Cullen, ${ }^{1} \mathrm{CR}$ Brooks, ${ }^{4} \mathrm{~J}$ Benschop, ${ }^{1} \mathrm{~A}$ 't Mannetje, ${ }^{5} \mathrm{R}$ Weinkove, ${ }^{1} \mathrm{~J}$ Douwes, ${ }^{6}$ NE Pearce. ${ }^{1}$ Centre for Public Health Research, Massey University, Wellington, New Zealand; ${ }^{2}$ Ecole Nationale Veterinaire, Agroalimentation et de I'Alimentation, ONIRIS, Nantes, France; '3nstitute for Environmental Science and Research, Upper Hutt, New Zealand; ${ }^{4}$ Institute of Veterinary, Animal and Biomedical Sciences, Massey University, Palmerston North, New Zealand; ${ }^{5}$ Capital and Coast District Health Board and Malaghan Institute, Wellington, New Zealand; ${ }^{6}$ London School of Hygiene and Tropical Medicine, London, UK

\subsection{6/oemed-2018-ICOHabstracts.605}

Introduction Leptospirosis is the most common occupational zoonosis in New Zealand, with the highest incidence observed in meat processing workers and farm workers. New Zealand has a high incidence of human infection relative to other temperate developed countries, and the organism is widespread in livestock. Serological testing has confirmed infection in livestock presenting to abattoirs and in meat workers. The objective of this study was to determine whether leptospires were present in bioaerosols within the abattoir.

Methods Ambient air samples $(n=18)$ were collected in an abattoir from ovine and bovine processing areas, using a SASS 3100 high volume sampler located adjacent to workers performing exsanguination (halal sticking), pelt removal, evisceration, a splitting saw (bovine only) and boning or meat cutting. Nucleic acid (DNA) in the bioaerosol samples was amplified using multiple displacement amplification (MDA) for metagenomic analysis, but the material was also tested for specific pathogenic species including $L$. interrogans sv Pomona and $L$. borgpetersenii sv Hardjobovis by quantitative PCR. The original (unamplified) DNA samples were also tested.

Result Leptospires were detected in 11 of the (MDA) samples from both ovine and bovine processing areas at the splitting saw, evisceration, exsanguination and pelt removal. There was no evidence of leptospires in samples taken in the boning or meat cutting areas, or in the five blanks taken. Two of the original DNA samples, both from the ovine pelt removal area, also tested positive for leptospires.

Discussion This is the first study to show that leptospires can be detected in a bioaerosol within an abattoir, suggesting a possible route of transmission to meat workers. The organism was detected at locations adjacent to slaughter, pelt removal and evisceration, with the strongest evidence near ovine pelt removal. This distribution directly mirrors the pattern of risk shown in serological testing of meat workers.

\section{Integrated Worker Health and Well Being}

\section{DEVELOPING HEALTHY WORKPLACES IN IRELAND}

Biddy O'Neill*. Health and Wellbeing Programme, Department of Health, Dublin, Ireland

\subsection{6/oemed-2018-ICOHabstracts.606}

Aim of special session To outline key elements in the development of the Healthy Workplace Framework and present Irish worksite case study

Mr. Robert F. Murphy ${ }^{1}$, Dr. Helen $\mathrm{McAvoy}^{2}$, Dr. Margaret Hodgins $^{3}$, Ms. Una Feeney ${ }^{4}$ 